\title{
Multiboson measurements (not photon-induced) at CMS
}

\author{
Alicia Calderon on behalf of the CMS Collaboration. ${ }^{a,}$ \\ ${ }^{a}$ Instituto de Fisica de Cantabria (CSIC - University of Cantabria), \\ Santander, Spain \\ E-mail: alicia.calderon@cern.ch
}

We present the most recent studies of multiboson production (not photon-induced) physics in proton-proton collisions at $13 \mathrm{TeV}$ center-of-mass energy based on data recorded by the CMS detector at the LHC in 2016-2018. These include precise measurements of WW, WZ, ZZ and the first observation of tribosons (VVV) production. Results on dibosons production at $5.02 \mathrm{TeV}$ center-of-mass energy are also presented using data collected by CMS in a special LHC run. Results include total and fiducial cross sections, as well as normalized differential cross sections in the fiducial region. These are compared to the most accurate theoretical predictions to the date. We also present the first observation of single longitudinally polarized $\mathrm{W}$ bosons in the WZ production.

The Ninth Annual Conference on Large Hadron Collider Physics - LHCP2021

7-12 June 2021

Online

${ }^{*}$ Speaker 


\section{Introduction}

The multiboson production processes are expected to be produced at the LHC and their cross sections, as well as detailed kinematic differential distributions, are precisely predicted in the framework of the Standard Model (SM). The cross section measurements provide excellent tests of the Quantum Cromodynamics (QCD) and the Electroweak (EW) sectors. Furthermore, the multiboson processes are themselves sources of background to the SM Higgs boson analyses, as well as searches for new phenomena. Moreover, the measurement of multiboson production cross section and kinematics provides direct information on trilinear and quartic gauge couplings (TGC and QGC). The SM predicts precisely the possible self-interactions of gauge bosons, and any deviation would be attributable to anomalous couplings (aTGC, aQGC), indicating the presence of new physics.

\section{Measurement of $\mathrm{WW}, \mathrm{WZ}$ and $\mathrm{ZZ}$ production at $13 \mathrm{TeV}$}

The diboson processes presented here have been measured in the final charged lepton (electron or muon) decay channels and selections have been optimized independently in each case to get the maximum signal sensitivity. In case of the WW analysis [1], we required two isolated opposite charge leptons with different or same lepton flavour, while in the WZ analysis [2], we required exactly three isolated leptons with a pair of them of same flavor and having an invariant mass close to the mass of $\mathrm{Z}$ boson. In addition, significant missing transverse energy from $\mathrm{W}$ boson decay is required in both processes. The $\mathrm{ZZ}$ production cross section [3] have been measured using the high purity four lepton $\left(\mathrm{ZZ} \rightarrow 212 \mathrm{l}^{\prime}\right)$ region and requiring the invariant mass of each pair of those leptons to be compatible with the $\mathrm{Z}$ boson mass. The corresponding total production cross sections measured are:

$$
\begin{aligned}
\sigma_{W W} & =117.6 \pm 1.4 \text { (stat. }) \pm 5.5 \text { (syst.) } \pm 1.9 \text { (theo.) } \pm 3.2 \text { (lumi) } \mathrm{pb} . \\
\sigma_{W Z} & =50.6 \pm 0.8 \text { (stat.) } \pm 1.5 \text { (syst.) } \pm 0.5 \text { (theo.) } \pm 1.1 \text { (lumi)pb. } \\
\sigma_{Z Z} & =17.4 \pm 0.3 \text { (stat.) } \pm 0.5 \text { (syst.) } \pm 0.4 \text { (theo.) } \pm 0.3 \text { (lumi)pb. }
\end{aligned}
$$

Experimental measurements of the total WW, WZ and ZZ production cross sections have reached the level of few \% uncertainty, with the Run2 data. The high precision reached allows to discriminate between next-to-leading-order (NLO) and next-to-next-to-leading-order (NNLO) in QCD predictions. Differential cross section distributions normalized to fiducial cross sections are also measured, as shown in Fig. 1, for the WW (top) and ZZ (bottom) processes. In case of the WW, results are well described by the NNLO theoretical predictions, while in case of the ZZ some differences can be observed as discussed in [3].

Since the LHC is a pp collider, the $\mathrm{W}^{+} \mathrm{Z}$ and $\mathrm{W}^{-} \mathrm{Z}$ cross sections are not equal. The ratio of $\mathrm{W}^{+} \mathrm{Z}$ to $\mathrm{W}^{-} \mathrm{Z}$ are shown in Fig. 2 (left). Results shows enough precision to be able to favor NNLO predictions over the NLO ones, and start to be sensitive to different Parton Distribution Functions (PDFs). The first observation of longitudinally polarized $\mathrm{W}$ bosons in $\mathrm{WZ}$ production is also reported, with an observed (expected) 5.6 (4.3) standard deviations. In Fig. 2 (right) the confidence regions in the $f_{L R}-f_{0}$ parameter plane for the $\mathrm{W}$ boson polarization is shown. 

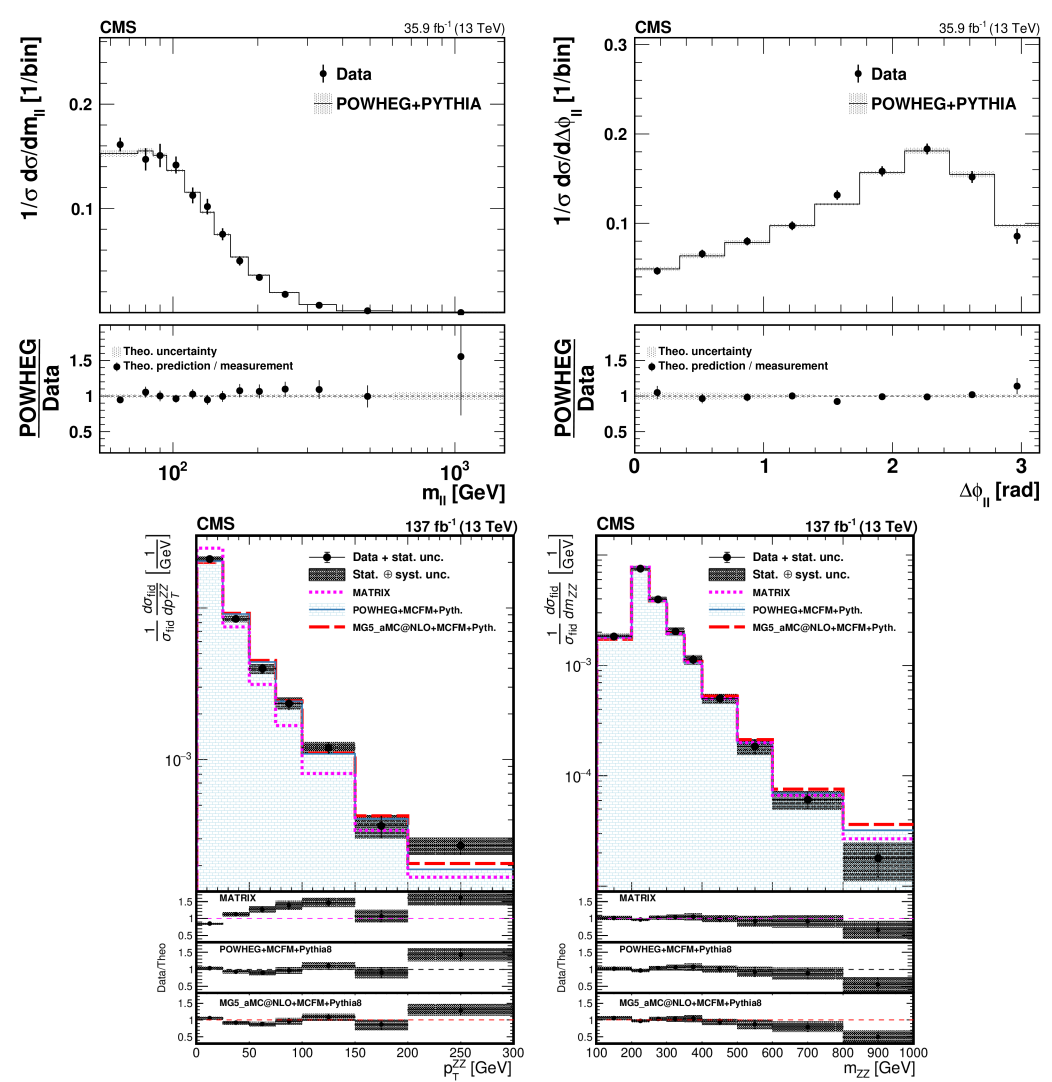

Figure 1: Top: Normalized differential cross sections for the WW process with respect to the dilepton invariant mass (left) and dilepton azimuthal angular separation (right). The grey bands in the ratio plots represent the systematic uncertainties, while the error bars on the markers represent the statistical uncertainties [1]. Bottom: Normalized differential cross sections for the $\mathrm{ZZ}$ process as a function of as a function of $p_{T}$ (left) and the invariant mass (right) of the ZZ system. The points represent the data with statistical uncertainties [3].

\section{Measurement of VV production at 5.02 TeV}

We report the first measurements of diboson production cross sections at a center-of-mass energy of $5.02 \mathrm{TeV}$ [4]. Events with two, three, or four charged leptons (electrons or muons) in the final state are analyzed. Results obtained in this analysis for the WW, WZ and ZZ diboson processes are shown Fig. 3, with other diboson production cross section measurements at different center-of-mass energies for the CMS, ATLAS, CDF and D0 collaborations, compared to the NNLO $\mathrm{QCD} \times$ NLO EW predictions from matrix. All measurements are in good agreement with NNLO QCD times NLO order electroweak cross section calculations.

\section{First observation of VVV production}

The first observation of the combined production of three massive gauge bosons (VVV with $\mathrm{V}$ $=\mathrm{W}, \mathrm{Z})$ at $13 \mathrm{TeV}$ is also reported here [5]. The measurements for individual WWW, WWZ, WZZ, and ZZZ production are performed in final states with three, four, five, and six leptons (electrons or 

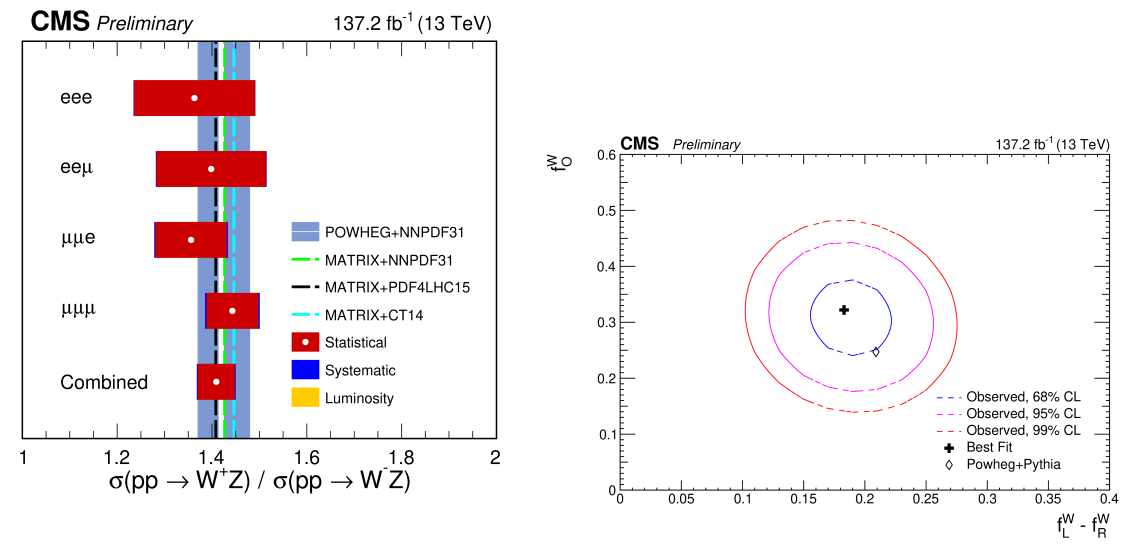

Figure 2: Left: Charge asymmetry ratio from the $\mathrm{WZ}$ analysis. The shaded vertical bands correspond to the the theoretical predictions from POWHEG (blue) and matrix (grey). Predictions obtained using matrix and several central replicas of different PDF sets are also shown. Right: Confidence regions in the $f_{L R}-f_{0}$ parameter plane for the $\mathrm{W}$ boson polarization in $\mathrm{WZ}$ events [2].

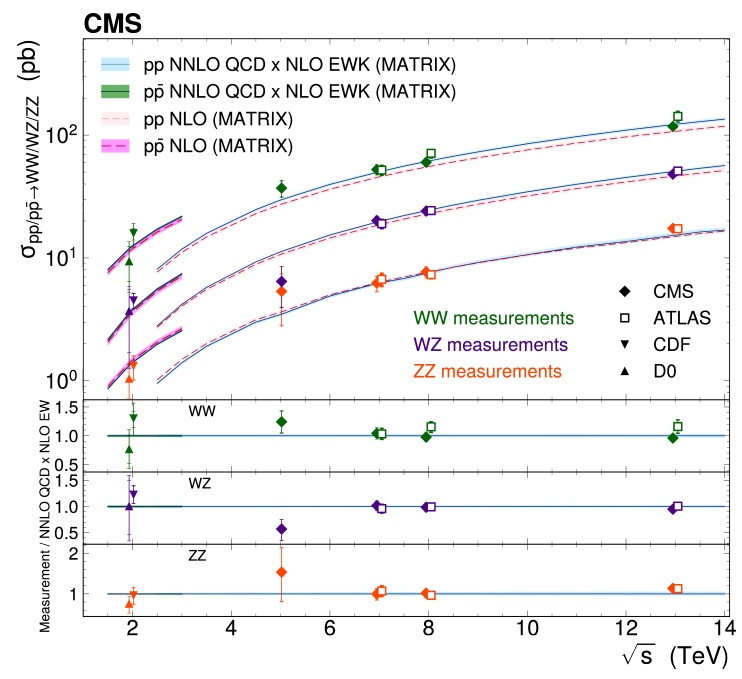

Figure 3: Results obtained for diboson production cross section measurements at $5.02 \mathrm{TeV}$. Also, different center-of-mass energies for the CMS, ATLAS, CDF and D0 collaborations are included, compared to the NNLO QCD $\times$ NLO EW predictions from matrix [4].

muons), or with two same-sign leptons plus one or two jets. The observed (expected) significance of the combined VVV production signal is 5.7 (5.9) standard deviations. The corresponding measured cross section relative to the standard model prediction is $1.02+0.26-0.23$. The significances of the individual WWW and WWZ production are 3.3 and 3.4 standard deviations, respectively. 


\section{References}

[1] CMS Collaboration, $W^{+} W^{-}$boson pair production in proton-proton collisions at $\sqrt{s}=$ 13 TeV, PhysRevD 102 (2020) 092001.

[2] CMS Collaboration, Measurement of the $\mathrm{pp} \rightarrow \mathrm{WZ}$ inclusive and differential cross sections, polarization angles and search for anomalous gauge couplings at $\sqrt{s}=13 \mathrm{TeV}, C M S-P A S$ SMP-20-014 (2021).

[3] CMS Collaboration, Measurements of $\mathrm{pp} \rightarrow \mathrm{ZZ}$ production cross sections and constraints on anomalous triple gauge couplings at $\sqrt{s}=13 \mathrm{TeV}$, Eur.Phys.J.C 81 (2021) 200.

[4] CMS Collaboration, Measurements of the weak diboson production cross sections in leptonic decays at $\sqrt{s}=5.02 \mathrm{TeV}$ with the CMS experiment, CMS-PAS-SMP-20-012 (2021).

[5] CMS Collaboration, Observation of the Production of Three Massive Gauge Bosons at $\sqrt{s}=$ 13 TeV, Phys. Rev. Lett. 125 (2020) 151802. 